\title{
NOTES ET ESSAIS \\ CONCERNANT LE NOURRISSAGE DE LA TRUITE
}

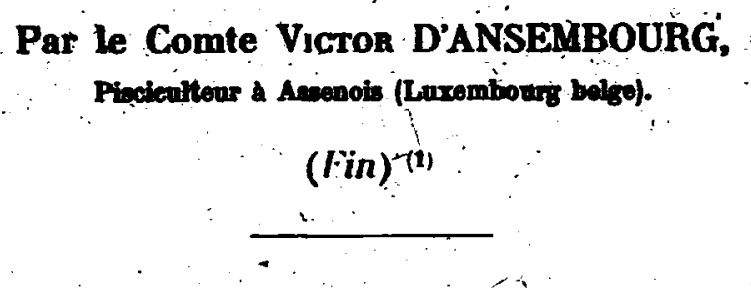

Au reste, voici, mis en tableau, le détail des rations quotidiennès proportionnelles et des quotients alimentaires absolus.

\begin{tabular}{|c|c|c|c|}
\hline$\cdots$ & -R.P. & Q.A. & OBSERVATIONS \\
\hline 25 Septembre au 27 Octobre & $1,6 \%$ & & ids total se main- \\
\hline 27 Octobré an 25 Novembre. & $6,8 \%$ & 7,2 & L'accroissement journa- \\
\hline $\begin{array}{l}25 \text { Novembre au } 28 \text { Décembre. . . } \\
28 \text { Décembre au } 3 \text { Mars. . . . . . }\end{array}$ & $\begin{array}{l}2,2 \% \\
1,5 \%\end{array}$ & 12,7 & Le poids total se main- \\
\hline 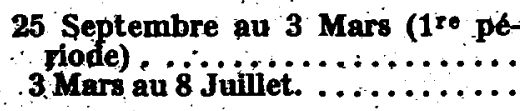 & $\begin{array}{l}2,5 \% \\
4,7 \%\end{array}$ & $\mathbf{3 2}^{\mathbf{1 3}, 7}$ & L'accroissement est très \\
\hline 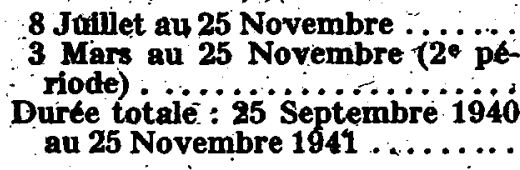 & $\begin{array}{l}6,2 \% \\
5,1 \% \\
3,26 \%\end{array}$ & $\begin{array}{l}15 \\
20 \\
16,4\end{array}$ & $\therefore$ \\
\hline
\end{tabular}

Nous constatons de prime abord une différence bien marquée entré la première période, qui comprènd l'automne et l'hiver, et la deuxième, qui englobe principalement le printemps et l'été. La première est nettegnent moins défavorable, malgré une R.P. beaucoup plus basse. Nous avons, d'une part, une R.P. de $2,5 \%$ qui produit un certain accroissement, et, d'autre part, une R.P. double $(5,1 \%)$; dont le rendement est plus faible. Cétte différence s'explique, par la température moyennẹ plus élevée, pour l'ensemble de la deuxièmè période, par. les exigences alimentaires pdus impérieuses et par une plus grande sensibilité vis-à-vis des jeunes et des a-courps du nourrissage. Et, dans notre expérience, l'approvisionnement, quoique plus abondant, fut encore plus ehaotique en été.

(1) Voir : - Bulletín, n॰ 126, Juillet-Septembre 1942, p. 25. 
En examinant le détail de la première période, nous voyons deux cas de stabilisation du poids. La ration d'entretien peut donc être fixée ici, avec une certaine précision, à $1,6 \%$, en Octobre et a $1,5 \%$ en hiver, les

- températures moyennes de l'eau étant respectivement de $11^{\circ} \mathrm{C}$. et de $8^{\circ}, \mathrm{C}$. environ.

Pour le printemps et le début de l'été, la R.E. ne ressort pas aussi nettement des chiffres, et nous devrons nous contenter d'une approximation. Elle est en tout cas située plus bas que 4,7\%, puisque la.R.P. égale à ce chiffre est légèrement productive. Admettons qu'elle se soit établie entre 4 et $4,5 \%$. Les mauvaises conditions du nourrissage interviennent sans doute plus que la température dans cetto grande différence avec la R.E. du mois d'Octobre, car, du 3 Mars au 8 Juillet, la température moyenne de notre eau n'est guère plus haute que celle de l'automne. Il est vrai, cependant, que les maxima sont plus élevés, en Juin, par exemple, et que le printemps se signale de toute facon par une recrudescence des appétits.

Les jeânes prolongés obligent l'organisme à consommer ses réserves pour couvrir ses besoins énergétiques, qui nè chôment pas. Ces prélèvements font baisser le poids du corps, et la quantité de nourriture nécessaire pour rattraper les kilos perdus est vraisemblablement plus grande que celle qui aurait assuré l'entretien de ce poids, s'il y avait eu plus de continuité dans le nourrissage. Il est donc permis d'admettre que, si les distributions de la même masse d'aliments avaient pu être régulières et fréquentes, la croissance aurait été plus forte, et la ration d'entretien sé serait établie un peu plus bas qu'a $1,5=$ en hiver, et bien au-dessous de $4,5 \%$ au printemps et au début de l'été, car l'irrégularité de l'approvisionnement fut, comme nous l'avons dit, encore plus marquée pendant la deuxième période que pendant la première.

- Ajoutons un facteur qui aurait agi dans le mềme sens : si nợs avions considéré, au lieu du poids brut de. la nourriture; le poids net des rations effectivement données aux poissons, nous aurions également vu un rabaissèment des rationis d'entretien.

Somme toute, les points suivants nous semblent utiles à retenir pour les cas d'exploitation au ralenti :

$1^{\circ}$ La ration d'entretien est relativement minime à partir du mois d'Octobre jusqu'au début du mois de Mars. En cas de production ralentio, il est donc plus économique d'entretenir un poids donné de Truites en automne et en hiver qu'au printemps et qu'en été.

$2^{\circ}$ A égalité de température moyenne de l'eau, la ration d'entretien parait plus basse en automne qu'au printemps.

$3^{\circ}$ L'irrégulaxité de l'approvisionnement et les longues périodes de jeane doivent provoquer, surtout au printemps et en été, une forte hausse de la R.E. et par conséquent de grands gaspillages de nourriture.

$4^{\circ}$ La conclusion de Barthílemy et Mugard (voir plus loin), concernant 
l'emploi renforcé de matières végétales, peut trouver place ici, car elle intéresse les exploitations paralysées.

Les cas anormaux, " pathologiques ", ont ceci de bon qu'ils contiennent presque toujours un enseignement. Dans cette expérience vécue, nous avons cru voir quelques lueurs sur une question importante el relativement peu fouillée. C'est pourquoi nous la sortons de l'ombre.

\section{2. - Variations de la ration d'entretien.}

Les bésoins énergétiques et, par.conséquent, la ration d'entretien varie: " avec toute une série de facteurs : température, oxygène dissous daris l'eau, mouvements des poissons, ou, si l'on veut, dimensions des bassins, genre de nourriture, relation nutritive, fréquence des repas, taille, âge, espèce, individualité, etc. Nous nous en tiendrons à quelques-uns d'entre eux.

La température et l'oxygène dissous agissent très prófondément sur la R.P. et sur la R.E. Les échanges énergétiques ne consomment pas seulement des hydrates de carbone, de la graisse et même, comme nous le verrons, de la protéine, mais encore de l'oxygène. L'entretien du corps vivant est donc le fait, naturellement, de l'alimentation; et aussi, on n'y songe peut-être point assez, de la respiration.

L'oxygène assure les phénomènes d'oxydation qui produisent l'énergie,dont le résidu se manifeste sous formé de chaleur. Le maintíen d'une température constante chez les animaux à sang chaud exige donc- une nourriture plus riche, plus calorigène, lorsqu'il fait froid. Cette plus grande quantité d'aliments réclame à son tour plus d'oxygène pour la " bruler ". On sait que, chez les animaux à sang froid, c'est le contraire qui se produit. En hiver, leurs échanges intra-organiques sont ralentis; ils mangent moins et consomment moins d'oxygène qu'en été. Une élévation de température de $10^{\circ} \mathrm{C}$ double à peu près leurs échanges énergétiques (cette accélération diminue dans les températures élevées, à partir de $20^{\circ} \mathrm{C}$ environ, pour la Truite). Il s'ensuit que, si la R:E. est par exemple de $1,5 \%$ a $6^{\circ} \mathrm{C}$, elle approchera de $3 \%$ a $16^{\circ} \mathrm{C}$.

Les animaux à sang froid ont toutefọis ceci de commun avec les homéothermes, que leurs besoins énergétiques sont proportionnels a leurs activités externes et internes et à la surface de leur corps. Retenons cette question de surface. C'est elle; principalemènt, qui détermine en réalité les inffluences sur la R.P. attribuées à la taille; au poids ou à l'âge. C'est pour la facilité que la R.P. et la R.E. se calculent en fonction du poids : de fait; la surface y entre bien plus en jeu que le poids.

On peut se faire une idée de l'importance de ce facteur si l'on songe auxx différences considérables qui existent entre les surfaces de deux volumes égaux divisés, l'un, en petits volumes, l'autre, en volumes plus grands. Ainsi, la surface d'un kilo d'alevins pesant individuellement un gramme est cinq fois plus grande que celle d'un kilo de Truites de 125 grammes. 
S'il s'agit d'alevins venant d'éclore et pesant 0,1 gramme, leur̀ surface à poids égal est dix fois plus étendue que celle de Truites de 100 grammes, dont le poids individuel est mille fois plus grand.

Remarquons en passant que 5 et 10 sont les racines cubiques de 125 et de 1000. Les besoins alimentaires (R.P. et R.E.). et respiratoires des alevins sont donc proportionnellement beaucoup plus élevés que ceux des Truites de taille supérieure.

Si le rôle de la surface paratt moins étroitement lié aux pertes de chaleur par rayonnement, chez les animaux à sang froid que chez les homéothermes, qui sont obligés de maintenir leur corps à une température habituellement plus élevée que celle du milieu extérieur, il n'en semble pas moins vrai que ce rôle s'accrôt du fait d'une amplitude bien plus étendue des variations de là surface chez les Poissons que chez les Vertébọ́s supérieurs, el cela à cause des différences de taille plus accentuées. Une Truite de cent grammes pèse mille fois plus qu'un alevin fratchement éclos - et elle n'est pas au bout de sa croissance -, tandis que le poids d'un homme adulte n'est que vingt à vingt-cinq fois plus grand que celui d'un nouveauné

\section{3. - Principes nutritifs entrant dans la ration d'entretien.}

On sait que l'entretien du corps incombe aux hydrates de carbone et aux matières grasses, tandis que les matières albuminoides, ou, si l'on veut, la pnotéine - indispensables à la formation du muscle - se chargent plutôt de Ia croissance.

Cette spécialisation des tâches imparties aux divers composants de la ration n'est pas absolue. En vertu de la loi de l'isodynamie des principes nutritifs, ceux-ci sont tous capables d'assurer l'entretien, car ils sont tous endothermiques et contiennent donc de l'energie et de la chaleur latentes, que l'oxydation dégage. Ils ont également le pouvoir de faire de la graisse.

Ainsi, les hydrates de carbone, les matières grasses, et, à leur défaut, ou lorsqu'il y a excès de proténe, la protéine elle-mềme, peuvent indifféremment produire de l'énergie, de la chaleur et de la graisse de réserve. Mais l'inverse $n$ 'est pas vrai : les matières non azotées seront toujours inaptes a fournir l'élément essentiel de lá croissance, car celui-cị est précisément l'azote qui-leur manque.

Si là protéine est à même de couvrir les besoing énergétiques de l'organisme, son prix, toujours plus élevé que celui des matières hon azotées, doit la faire reléguer à l'arrière-plan dans ce rôle impróductif: Cette règle, importante dans l'alimentation des animaux domestiques, semble pouvoir trouver une application atténuée en trutticulture.

Bien entendu, il n'est pas question de supprimer les albuminoìdes, ni. même de les réduire autant qu'il serait permis de le faire pour des animaux qui exigent une relation nutritive lache, et chez qui l'entretien sera assuré presque uniquement par les matières non azotées, tels que la Carpe, 
le bétail, etc. L'estomac de la Truite, spécialisé en vertu de la pepsine, son ferment digestif, dans l'élaboratiqn de la protéine, ne supporterait pas ce régime: Mais il est bon de songer à économiser les albuminoïdes et à expérimenter jusqu'à quel point une relation nutritive, spécifiquement très étroite, peut être élargie par un renforcement de la dose des hydrates de carbone. Nous ne parlons pas des matières grasses, car on sait que la Truite en tolère fort peu.

-En fournissant un complément de matières non azotées, on assure une meilleure répartition des tâches et l'on fait, somme toute, de la rationalisation, s'il est permis d'employer ce terme-quand il s'agit de rétablir un équilibre rompu par l'intervention de l'homme. En effet, la nature, dont nous croyons peut-être violer ainsi les lois, et spécialement celle qui préside à l'alimentation des carnivores, nous devance bel et bien dans cette voie.

La Truite est carnivore, c'est entendu ; mais il ne faudrait pas croire pour cela qu'il convient de la nourrir uniquement de viande et de poisson. En régime naturel, elle s'offre toujours des animaux entiers, le plus souvent herbivores, qu'elle avale avec le contenu végétal de leur estómac et de leur intestin, avec leurs ferments digestifs, leur chitine, leur coquille, leur fourreau de graviers ou de bois, toutes choses que la viande morte ne contient pas ou ne contient plus, qui fournissent à la mangeuse des hyḍates de carbone, des. vitamines et des matériaux de bourrage, et que le nourrissage doit tenter de remplacer.

Il est donc logique de compléter la ration par des matières végétales. Si ce complément doit être faible en temps d'exploịtation à plein rendement, il peut théoriquement être augmenté lorsqu'on est obligé de travailler au ralenti.

Partant de ce principe, Banthílemy et Musaro (1) ont fait deux intéressantes expériences dont le but était, précisément, de “ remplacer pour les dépenses énergétiques les albuminoỉdes côteuses par des hydrates de corbone relativement moins chers ". La réalisation pratique donna raison ¿ la théorie, car il ressort du résultat obtenu qu'une certaine quantité de blé, jointe à une ration de farine de poissons qui - nous le savons par comparaison - ne couvrait pas tout à fait lés besoins d'entretien, a provoqué un accroissement journalier égál à près de $0,7 \%$ du poids initial des Truites. Sans le blé, il est à peu près certain qu'il n'y aurait pas eu la moindre croissance.

L'appoint végétal, s'il n'a pas joué un rôle direct dans cette croissance, a du moins permis à une partie de la protéine, en la délivrant d'une tâche qui ne lui incombe pas, d'accomplir sa besogne constructive. G'est un bon exemple d'expérience raisonnée et récompensée par un résultat concret.

(1) Loc. cif., pp. 290-294. 
Qu'il nous soit permis, pour terminer, de suggérer un essai. Il ne serait pas très compliqué de déterminer expérimentalement la ration d'entretien qui prévaut dans tel ou tel cas. Il suffirait d'isoler quelques lots de Truites et de les soumettre à des conditions identiques, sauf en ce qui concerne les quantités de nourriture. Bien entendu, les températures et les débits de l'eau seraient soigneusement relevés. Chaque lot recevrait journellement : le premier, une ration égale à $1 \%$ de son poids, le deuxième, une ration de $2 \%$, le troisième, une ration de $3 \%$, et ainsi de suite. Une première période d'un mois montrant, par exemple, que le poids des sujets décrołt si la R.P. est de $2 \%$, et qu'il croft si celle-ci est de $3 \%$, on continuerait l'expérience autant que possible à la même température d'eau, en utilisant des R.P. comprises entre $2 \%$ et $3 \%$, et ainsi, par approximations successives, la part improductive de la ration serait fixée aussi exactement qu'on peut le souhaiter.

Ceux qui en auraient le temps et les possibilités approfondiraient ces recherches en faisant varier certains facteurs : le genre de nourritüre, la relation nutritive, la fréquence des repas, l'espèce (Truite arc-en-ciel ou fario), l'âge, la taille, etc., etc.

Evidemment, c'est un programmè chargé. $\mathrm{Si}$, lorsqu'ils le pourront, quelques praticiens exécutaient ne fât-ce qu'une expérience, ou bien deux, l'une en été, l'autre en hiver, en employant leurs aliments habituels, les résultats seraient déjà précieux pour ces expérimentateurs et intéressants pour leurs confrères. 